\title{
Non-Invasive Glucose Monitoring: Application and Technologies
}

\author{
Ghozzi Dorsaf ${ }^{1}$, Manai Yacine ${ }^{1 *}$ and Nouri Khaled ${ }^{2}$ \\ ${ }^{1}$ Laboratoire de Recherche des systèmes Avancés, Ecole Polytechnique de Tunisie, Tunisie \\ ${ }^{2}$ Laboratoire de Recherche des systèmes électrique, école national d'ingénieurs de, Tunise
}

Submission: January 23, 2018; Published: April 13, 2018

*Corresponding author: Manai Yacine, Laboratoire de Recherche des systèmes Avancés, Ecole Polytechnique de, Tunisie, Email: manai@gmail.com

\begin{abstract}
The chronic metabolic disorder, diabetes mellitus is a fast-growing global problem with huge social, health, and economic consequences. The prevailing method for determination of blood glucose concentration is using a self-monitoring glucose meter. Most of the commercially available devices for glucose measurement are invasive or minimally invasive. Invasive devices used for blood glucose monitoring are painful whereas minimal invasive devices have limited time span and stability. Thus, there is a need of an economic, compact, painless and convenient noninvasive device which can promote frequent blood testing which help in control of blood glucose level. There have been numerous developments in the field of non-invasive glucose monitoring NGM during the last decade, which stress the need for a critical review. This manuscript aims to review the various NGM techniques and application. The limits and future trends in NGM are also discussed.
\end{abstract}

Keywords: Non-invasive glucose monitoring; Diabetes; Technology; Application

\section{Introduction}

The prevalence of diabetes is constantly increasing worldwide at an alarming rate. According to the International Diabetes Federation in 2015, an estimated 415 million people globally were suffering from this condition [1]. Currently no treatment exists or is under development which could possibly cure this illness in the near future. The therapy of diabetes mellitus so far consists in monitoring the blood glucose (BG) level of a patient to avoid the danger of hypo- and hyperglycemias and to assist in adjusting the diet and medical treatment.

Self-monitoring of BG concentrations has advanced over the past few years. Glucose values determined by home meters correlate well with laboratory results. Because of the importance of precision and accuracy of self-monitoring $B G$ devices, guidelines for the performance of these devices were recommended in 1987 by the American Diabetes Association [2-5].

Almost all commercial blood glucose monitoring devices (BGMD) employ a cost-effective electrochemical biosensor, which is capable of being mass produced and responding rapidly to glucose detection. They use automatic lanced devices to prick the fingertip of diabetics has to measure blood glucose very frequently. There have been tremendous developments in the last few decades to develop improved BGMD [6] with reduced blood sample requirement of less than $1 \mu \mathrm{L}$. The painful aspect are minimized by employing alternate sampling sites (hand, arm). However, the cost of strip and the boredom of making repeated measurement are becoming most important. The minimally invasive approaches have been developed by using subcutaneous sensors to determine glucose concentration in interstitial fluid. But they suffer from limitations in terms of discomfort to patients, requirement of continuous calibration, and high susceptibility to biofouling. Therefore, the development of NGM techniques $[7,8]$ is the only way to develop pain-free glucose monitoring technology for diabetics. This is the major stimulant for continuous ongoing developments in the field of NGM. There have been continuously increasing number of publications in NGM (Figure 1).

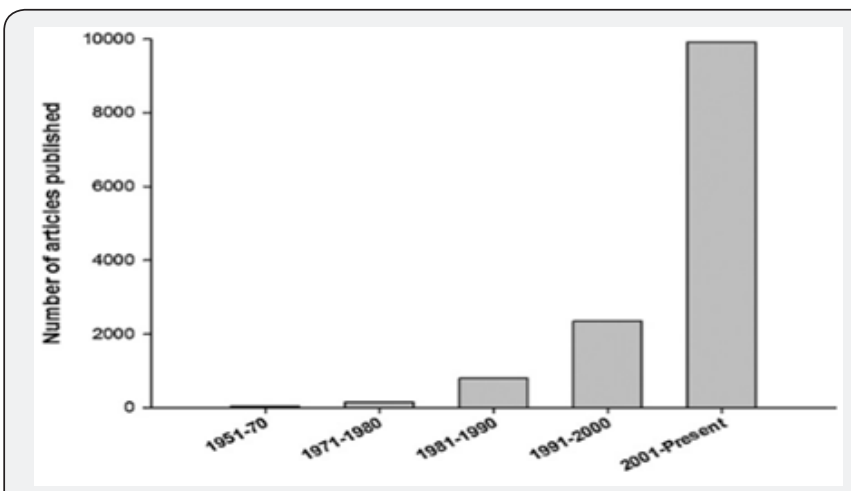

Figure 1: Number of articles pertaining to non-invasive glucose monitoring published during the mentioned period. 
The developments undertake to improve the way of measuring the BG depend on several parameters such as the reading accuracy, techniques and approach, size of the device, and technology of the embedded systems. Each of this generation will be discussed [9-11].

The recent development of the BGMD is more toward noninvasive method. This method is used to measure BG levels in the human body by only placing the sensors directly to the human targeted area without drawing blood and insertion of needles or any types of biosensors [12-16]. The study on the development of this technology has begun since 1957 and the works are still continuing up to the present. Since 2014, only a few numbers of devices that have been produced using this technology and they are only available in certain countries. Most of the noninvasive BGMD is registered under consumer product and not under a medical product because this device reads the value of the blood glucose without directly in contact with the blood [17-20].

This manuscript provides an update of various potential NGM technologies and devices along with their advantages, limitations. The goal of this study is to provide an update of various potential NGM technologies and devices along with their advantages and limits. In section II non-invasive glucose monitoring technologies have been discussed, while section III discusses the proposed NGM application, and section IV discusses technology vesus application. Conclusions and future developments have been illustrated in section V.

\section{Non-Invasive Glucose Monitoring: Technologies}

Non-invasive methods for glucose monitoring are more desirable and excellent alternatives to the above mentioned technologies [21]. This could make millions of people more relaxed and comfortable about regular blood glucose testing. Although a large number of research works have already been carried out in this area, the search of a successful non-invasive technique still continues [22].

The order of presentation is partially arbitrary: we tried to report first those technologies more known, used and described.

\section{Near infrared spectroscopic}

It is a spectroscopic method which uses radiation in the near infrared (NIR) region of the electromagnetic spectrum (750$2500 \mathrm{~nm}$ ) [6-7]. There are mainly three bands in NIR range :combination overtone band (2000-2500nm), first overtone band (1400-2000nm) and second or higher overtone band (750$1400 \mathrm{~nm})$. It allows the glucose measurement under the skin to a depth of few $\mathrm{mm}$ in range. The penetration of light into the skin decreases with the increase in wavelength [8]. As the light interacts with tissue, it is partially absorbed and scattered due to interaction with chromophores within the tissue [9]. The combination and the first overtone regions are dominated by glucose absorption information while shorter wavelength region mainly carries scattering information [10].

NIR light transmission or reflectance has been studied through an ear lobe, finger web and finger cuticle, skin of the forearm, lip mucosa, oral mucosa, tongue, nasal septum, cheek, and arm. NIR diffuse reflectance measurements performed on the finger showed a correlation with BG but predictions were often not sufficiently accurate to be clinically acceptable [11].

The technique has serious limitations as it is affected by physicochemical parameters such as changes in body temperature, blood pressure, skin hydration, and concentrations of triglyceride and albumin. Moreover, it is sensitive to environmental variation in temperature, humidity, atmospheric pressure and carbon dioxide content. The measurement are also affected by the thickness and thermal properties of the skin [12-13], and the disease states such as hyperglycaemia and hyperinsulinemia [14-15].

\section{Mid-infrared spectroscopy}

Mid-infrared (Mid-IR) spectroscopy is based on light in the $2500-10.000 \mathrm{~nm}$ spectrums [16]. The physical principle is similar to that of NIR. When compared to NIR, however, due to the higher wavelengths, Mid-IR exhibits decreased scattering phenomena and increased absorption. For this reason, the tissue penetration of light can reach a few micrometers [17]: in the case of human skin, that corresponds to the stratum corneum. As a consequence, only reflected, scattered light can be considered: there is no light transmitted through a body segment. On the other hand, a possible advantage of Mid-IR compared to NIR is that the Mid-NIR bands produced by glucose, as well as other compounds, are shaper than those of NIR, which are often broad and weak.

Mid-NIR is less studied technique compared to NIR for glucose measurement, probably due to the strong limitation in penetration. Studies are reported related to finger skin and oral mucosa [18]. One strong limitation is the poor penetration. Furthermore, Mid-IR is a affected by similar problems and confounding factors than NIR, despite glucose bands potentially improved for instance, some studies have shown significant dependence of skin Mid-IR spectrum on its water content [17].

\section{Raman spectroscopy}

Raman spectroscopy used laser radiation source from visible to Mid-IR, when some portion of scattered light shows a different wavelength from that of excitation beam, the effect is known as Raman effect. When scattered light has a higher wavelength and lower intensity (10-3 times) than the original signal shown in Figure 2. 


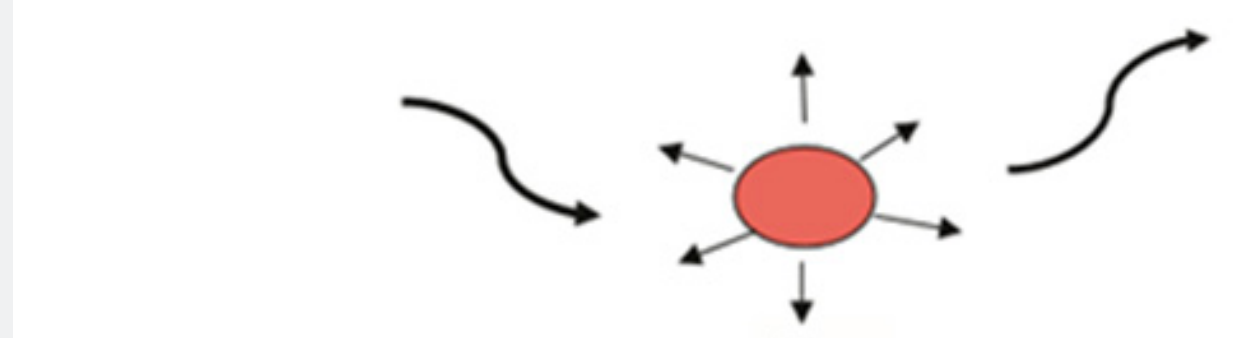

(a)

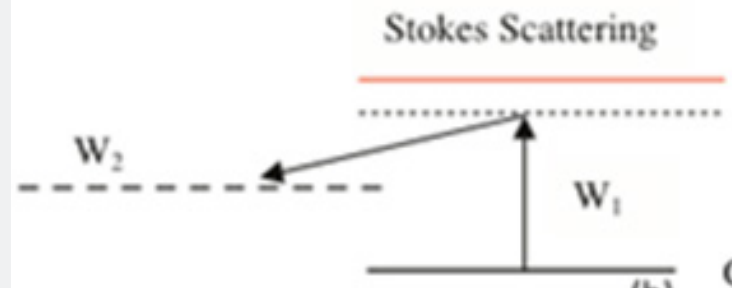

(b)

Ground State

Anti Stokes Scattering

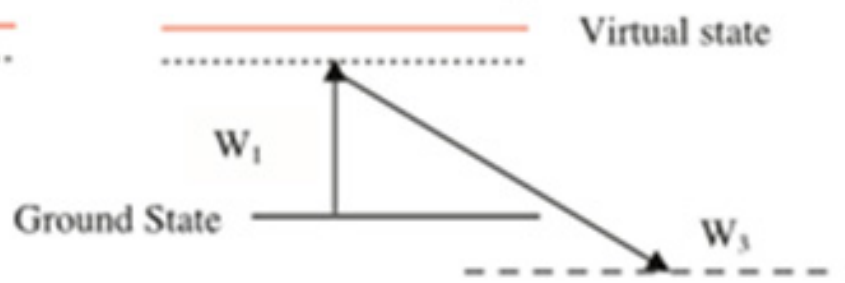

Figure 2: Basic principle of Raman spectroscopy: (a) a small portion of photons incident on a target molecule scatters with a shift in photon energy due to molecul vibration, (b) energy diagram representing the process of scattering.

\section{Optical coherence tomography}

The optical coherence tomography (OCT) allows the real time and precise non-invasive glucose monitoring. OCT uses low coherence light source, an interferometer and a photo-detector to measure the interferometric signal. The interferometer consists of reference arm, sample arm and a moving mirrors. Light returned from the reference part of the interferometer is combined with the backscattered light from tissue. The resulting interferometric signal is detected by a photo-detector. The delay in back scattered light or the changes in scattered light due to variation in glucose concentration is used for measurement of blood glucose [19-20].

\section{Bioimpedance spectroscopy}

The first study of non-invasive continuous glucose monitoring system involving impedance spectroscopy was published by Cadduff's group in 2003. As result from this research, the company pendragon developed a wrist glucose monitor called Pendra. The equipment gatherers information of a LC resonance circuit from $1 \mathrm{MHz}$ until $200 \mathrm{MHz}$, with the skin working as dielectric from the capacitor. One limitation of this research is that it requires an equilibration process, where the patient must rest for 60 min before starting measurement [22].

\section{Fluorescence spectroscopy}

This technique is based on the generation of florescence by human tissues when excited by lights at specific frequencies. In the case of glucose, one study demonstrated that when a glucose solution is excited by an ultraviolet laser light at $380 \mathrm{~nm}$, fluorescence can be detected at 340,380,400 nm, with maximum at $380 \mathrm{~nm}$ [16]. It was also proved that fluorescence intensity was dependent upon glucose concentration in the solution. Also light in the visible spectrum can be used, but this is more adequate for studying fluorescence of tissues rather than that of solutions. In tissues, the use of ultraviolet light could lead to strong scattering phenomena, in addition to fluorescence. Moreover, even when using different wavelengths, the fluorescence phenomenon can depend not only on glucose, but on several parameters, such as skin pigmentation, redness, epidermal thickness [21].

\section{Non-Invasive Glucose Monitoring: Application}

\section{GlucoWatch ${ }^{\circledR}$}

The first commercial U.S. Food and Drug Administration (FDA) approved non-invasive glucose monitor was the GlucoWatch ${ }^{\circledR}$ biographer (Cygnus Inc.). This wrist worn system electrochemically measured glucose concentrations in skin interstitial fluid (ISF) extracted by reverse iontophoresis (RI) [23-24]. The GluoWatch ${ }^{\circledR}$ electrochemically detected levels of extracted ISF glucose through enzymatic glucose oxidation at skin-worn sensing electrodes modified with glucose oxidase (GOx). The GlucoWatch ${ }^{\circledR}$ had a user-friendly interface that included an alarm for greater than 35\% changes in glucose, an activity marker (for meals, exercise, and insulin injection), accompanying software for data analysis and internal memory to store up to 8500 readings [24-25]. Clinical trials of the GlucoWatch ${ }^{\circledR}$ showed adequate precision for home bloodglucose monitoring. The Cygnus GlucoWatch was marketed in the early 2000s but was retracted from the market due to various reasons, which included reported skin irritation caused by the RI process, the long necessary warm up time (2-3h), and the need for calibration using standard blood glucose strips. To address these drawbacks, recent research efforts have focused on producing reliable, efficient non-invasive glucose monitoring platforms. 


\section{Tattoo-based glucose sensor}

Wang et al. recently developed a wearable, tattoo-based non-invasive glucose monitoring platform based on integrating RI with amperometric glucose detection on a flexible substrate [26]. The iontophoretic and glucose sensing electrodes were fabricated on a single temporary tattoo platform using screenprinting, leading to a body-compliant and easy to wear flexible, conformal device for single use glucose measurements. This sensing platform obviated the discomfort of Glucowatch ${ }^{\circledR}$ by reducing the applied iontophoretic current for ISF extraction and by utilizing a low voltage cathodic detection of the GOxgenerated hydrogen peroxide at the Prussian Blue electrode transducer. The performance of the tattoo-based electrodes was evaluated with healthy human subjects by comparing the recorded glucose signals before and after meal, and the results were validated by simultaneous blood glucose measurement using a commercial glucometer. Control experiments were carried out without enzyme modification of the glucose sensor to confirm the sensing mechanism. Although the tattoo-based device was intended for disposable use, such a sensor holds considerable promise for continuous non-invasive ISF glucose monitoring by offering a body-compliant, flexible and costeffective platform. Future work toward the development of such systems should focus on the testing of performance over long-term use, sensor integration with wireless electronics, and examination with large populations of both healthy and diabetes suffering individuals.

\section{Non-invasive sweat glucose monitoring}

Sweat is a very attractive bio-fluid toward non-invasive, continuous monitoring applications due to its distinct advantages, such as having the most sampling sites outside the body, continuous access, an ease of collection device placement and comfort, as well as its composition of physiologically important electrolytes and metabolites [27]. Gao et al. [28] demonstrated a flexible and fully integrated sensor array platform for multiplexed in situ perspiration analysis, measuring multiple sweat metabolites (glucose and lactate) and electrolytes (sodium and potassium) as well as skin temperature in a wearable patch type platform. The reported device integrated signal transduction, processing and wireless transmission for the realization of a practical wearable sensor device.

\section{Pendra}

Pendra from pendragon medical ltd, Switzerland was another NGM device in the form of a wrist-watch, which based on impedance spectroscopy. It was approved by FDA in may, 2003 as an adjunctive device to standard blood glucose meters to detect trends and patterns in glucose levels. It consists of an open resonant circuit (1-200 MHz) that lies in contact with skin with the help of a tape on the backside of the device and performs the impedance measurements. It cans perform up to four measurements per minute with sensitivity in the range of $20-60 \mathrm{mg}$ dL-1 glucose per ohm. It has USB connectivity for downloading data to a PC, Pendra user software for data analysis.
The correlation studies showed a poor correlation of only 35.1\%. The production of Pendra was stopped as the company went bankrupt in 2005.

\section{Application Versus Technologies}

In this review, we presented a description of NGM. According to the indications of the health technology assessment (HTA) methodology, we carried out the analysis on the basic of clear and objective criteria. Some of the technologies have not been exploited in a device yet, while some others have led to a device at least in advanced prototype condition. It must be noted however, that currently only three devices have reached a regulatory approval for commercialization in United States and/or Europe.

Among these three, for different reasons two of them are not on the market anymore. The only available one is the GlucoWatch, and in any case it cannot completely substitute a traditional meter. Furthermore, GlucoWatch is reported to possibly have some drawbacks: in fact, it sometimes causes skin irritation, and it may be uncomfortable to use in the daily life of the patients.

The development of non-invasive glucose biosensors for diabetes management is just beginning. Despite promising recent advances, there yet remain numerous challenges to overcome for the successful implementation of non-invasive glucose monitoring technologies, combining accurate real-time glucose readings with long-term stability. In particular, largescale studies are required to critically assess the accuracy

A reliable means of efficient, selective fresh glucose uptake is crucial to the development of sweat-based monitoring systems. Further efforts should focus on accurate epidermal glucose measurements by combination with sensing of other physiological parameters $(\mathrm{pH}$, temperature, humidity) toward continuous calibration and improved accuracy and correlation with blood glucose level. Future efforts should also aim at simultaneous monitoring of additional diabetes related markers toward a more comprehensive array of information regarding real-time glucose metabolism. Further improvement in iontophoretic sweat stimulation is desired for controllable (consistent and reproducible) sweat generation without the need for exercise. Additional work should also focus on improved sweat collection, flow and replenishment in connection to epidermal flexible microfluidics devices. Overcoming these and other challenges will be crucial for the advancement of continuous epidermal glucose monitoring to realize its potential impact.

To achieve the targeted goals, a thorough understanding of all components of the non-invasive monitoring systems are required, including the applied technologies, skin physiology, device surface and material chemistries, and sensing electrochemistry as well as the interface of these systems. The successful realization of non-invasive glucose monitoring platforms require not only consideration of scientific problems, but also economic, legal and commercial concerns with the patient and physician education of key importance. We envision 
that through a combination of these technological advances with large scale studies, glucose concentrations can be reliably and noninvasively monitored with minimal discomfort to patients for widespread applications toward improved glycemic control and autonomous therapeutic interventions through integration with autonomous, closed-loop insulin delivery systems. Given the rapid recent progress, it seems that non-invasive glucose monitoring has only scratched the surface of its full diagnostic potential and is poised to significantly increase its impact on medicine.

\section{Conclusion}

The non-invasive devices have tremendous appeal and a market in excess of at least one billion dollar per year worldwide. However, the development of non-invasive devices has been hampered by unsubstantiated claims, which proved to be misleading if not false.

In conclusion, the problem of non-invasive glucose monitoring is currently not solved, and further efforts are still necessary to reach the goal of having a reliable and inexpensive device for the benefit of the diabetic patient.

\section{References}

1. International Diabetes Federation (2015) IDF Diabetes Atlas. $\left(7^{\text {th }}\right.$ edn.),Brussels, Belgium: International Diabetes Federation.

2. Colwell JA, Dudley JD, McDonald JM, Metz R, Raskin P, et al. (1987) Consensus statement on self-monitoring of blood glucose. Diabetes Care 10: 95-99.

3. Vashist SK, Zheng D, Al-Rubeaan K, Luong JH, Sheu FS (2001) Anal. Technology behind commercial devices for blood glucose monitoring in diabetes management: a review. Chim Acta 703: 124-136.

4. Tura A, Maran A, Pacini G (2007) Non-invasive glucose monitoring: assessment of technologies and devices according to quantitative criteria. Res Clin Pract 77: 16-40.

5. Ferrante do Amaral CE, Wolf B (2008) Current development in noninvasive glucose monitoring. Med Eng Phys 30: 541-549.

6. Ozaki Y (2012) Near-infrared spectroscopy-its versability in analytical chemistry. Anal Chem 28(6): 545-563.

7. Nelson LA, McCann JC, Loepke AW, Wu J, Ben Dor B, et al. (2006) Development and validation of a multiwavelength spatial domain near-infrared oximeter to detect cerebral hypoxia-ischemia. J Biomed 11(6): 064022.

8. Rabinovitch B, March WF, Adams RL (1982) Non-invasive glucose monitoring of the aqueous humor of the eye. Part I. Measurement of very small optical rotations, Diabetes Care 5: 254-258.

9. Tura A, Maran A, Pacini G (2007) Non-invasive glucose monitoring: assessment of technologies and devices according to quantitative criteria. Diabetes Res Clin Pract 77: 16-40.
10. Amerov AK, Chen J, Small W, Arnold MA (2005) Scattering and absorption effects in the determination of glucose in whole blood by near-infrared spectroscopy. Anal Chem 77(14): 4587-4594.

11. http://www.ieee.org/organizations/pubs/newsletters/leos/apr98/ overview.htm

12. Sibbald RG, Landolt SJ, Toth D (1996) Endocrinol Metab Clin Clin North Am 25: 463-472.

13. Yeh SJ, Khalil OS, Hanna CF, Kantor S, JBiomed Opt 8: 534-544.

14. Yki-Jarvinen H, Utriainen T (1998) Diabetologia 41: 369-379.

15. Mazarevica G, Freivalds T, Jurka A (2002) J Biomed Opt 7: 244-247.

16. Khalil OS (2004) Non-invasive glucose measurement technologies: an update from 1999 to the dawn of the new millennium, Diabetes Technol Ther 6: 660- 697 .

17. Brancaleon L, Bamberg MP, Sakamaki T, Kollias N (2001) Attenuated total reflection-Fourier transform infrared spectroscopy as a possible method to investigate biophysical parameters of stratum corneum in vivo. J Invest Dermatol 116: 380-386.

18. Heise HM, Marbach R (1998) Human oral mucosa studies with varying blood glucose concentration by non-invasive ATR-FT-IR-spectroscopy. Cell Mol Biol 44: 899-912.

19. Gabbay RA, Sivarajah S (2008) optical coherence tomography-based continuous noninvasive glucose monitoring in patients with diabetes. Diabetes Technol Ther 10(3): 188-193.

20. Kirill VL, Mohsen SE, Massoud M, Rinat OE (2002) Noninvasive blood glucose monitoring with optical coherence tomography. Diabetes Care 25: 2263-2267.

21. Sandby-Møller J, Poulsen T, Wulf HC (2003) Influence of epidermal thickness, pigmentation and redness on skin autofluorescence. Photochem Photobiol 77: 616-620.

22. Caduff A, Hirt E, Feldman Y, Ali Z, Heinemann L (2003) First human experiments with a novel non-invasive, non-optical continuous glucose monitoring system. Biosens Bioelectron 19: 209-217.

23. Ackerman N, Berner B, Biegajski J, Chen Q, Chen H, et al. (2000) Controlled Drug Delivery (ACS Symposium Series). 752: 273-282.

24. Tierney MJ, Tamada JA, Potts RO, Jovanovic L, Garg S, et al. (2001) Clinical evaluation of the GlucoWatch(R) Biographer: a continual, non-invasive glucose monitor for patients with diabetes. Biosens Bioelectron 16(9-12): 621-629.

25. Tierney MJ, Kim HL, Burns MD, Tamada JA, Potts RO (2000) Electroanalysis of glucose in transcutaneously extracted samples. Electroanal 12(9): 666-671.

26. Bandodkar AJ, Jia WZ, Yardimci C, Wang X, Ramirez J, et al. (2015) Tattoobased noninvasive glucose monitoring: A proof-of-concept study. Anal Chem 87(1): 394-398.

27. Heikenfeld J (2016) Non-invasive analyte access and sensing through eccrine sweat: Challenges and outlook circa 2016. Electroanal 28 (6): 1242-1249.

28. Gao W, Emaminejad S, Nyein HYY, Challa S, Chen K, et al. (2016) Fully integrated wearable sensor arrays for multiplexed in situ perspiration analysis. Nature 529(7587): 509-514. 
This work is licensed under Creative Commons Attribution 4.0 License

DOI: 10.19080/CTBEB.2018.14.555878
Your next submission with Juniper Publishers will reach you the below assets

- Quality Editorial service

- Swift Peer Review

- Reprints availability

- E-prints Service

- Manuscript Podcast for convenient understanding

- Global attainment for your research

- Manuscript accessibility in different formats ( Pdf, E-pub, Full Text, Audio)

- Unceasing customer service

Track the below URL for one-step submission https://juniperpublishers.com/online-submission.php 